\title{
Patient presentation and physician management of upper respiratory tract infections: a retrospective review of over 5 million primary clinic consultations in Hong Kong
}

Kenny Kung ${ }^{1}$, Carmen Ka Man Wong ${ }^{1,4^{*}}$, Samuel Yeung Shan Wong ${ }^{1}$, Augustine Lam², Christy Ka Yan Chan ${ }^{1}$, Sian Griffiths ${ }^{1}$ and Chris Butler ${ }^{3}$

\begin{abstract}
Background: Upper respiratory tract infection (URTI) has a significant healthcare burden worldwide. Considerable resources are consumed through health care consultations and prescribed treatment, despite evidence for little or no effect on recovery. Patterns of consultations and care including use of symptomatic medications and antibiotics for upper respiratory tract infections are poorly described.

Methods: We performed a retrospective review of computerized clinical data on patients presenting to all public primary care clinics in Hong Kong with symptoms of respiratory tract infections. International Classification of Primary care (ICPC)codes used to identify patients included otitis media (H71), streptococcal pharyngitis (R72), acute URTI (R74), acute sinusitis (R75), acute tonsillitis (R76), acute laryngitis (R77), and influenza (R80). Sociodemographic variables such as gender, age, chronic illness status, attendance date, type and duration of drug prescribed were also collected.

Results: Of the 5,529,755 primary care consultations for respiratory symptoms from 2005 to 2010, 98\% resulted in a prescription. Prescription patterns of symptomatic medication were largely similar across the 5 years. In 2010 the mean number of drugs prescribed per consultation was 3.2, of which the commonly prescribed medication were sedating antihistamines (79.9\%), analgesia (58.9\%), throat lozenges (40.4\%) and expectorant cough syrup (33.8\%). During the study period, there was an overall decline in antibiotic prescription (8.1\% to 5.1\%). However, in consultations where the given diagnosis was otitis media (H71), streptococcal pharyngitis (R72), acute sinusitis (R75) or acute laryngitis (R76), over 90\% resulted in antibiotic prescription.

Conclusion: There was a decline in overall antibiotic prescription over the study period. However, the use of antibiotics was high in some conditions e.g. otitis media and acute laryngitis a. Multiple symptomatic medications were given for upper respiratory tract infections. Further research is needed to develop clinical and patients directed interventions to reduce the number of prescriptions of symptomatic medications and antibiotics that could reduce costs for health care services and iatrogenic risk to patients.
\end{abstract}

Keywords: Upper respiratory tract infection, Primary care, Pharmacology

\footnotetext{
* Correspondence: carmenwong@cuhk.edu.hk

'Division of Family Medicine, School of Public Health \& Primary Care, Chinese

University of Hong Kong, Shatin, Hong Kong

${ }^{4}$ Jockey School of Public Health, Prince of Wales Hospital, Room 408, 32

Ngan Shing Street, Shatin, NT, Hong Kong

Full list of author information is available at the end of the article
} 


\section{Background}

Respiratory infections are the commonest health problem encountered in primary care worldwide [1-3], amounting to between $16 \%$ to over $60 \%$ of attendances depending on locality $[4,5]$. Upper respiratory tract infection (URTI) is an important cause of reduced activity days, school and work loss, impaired school performance, and increased healthcare utilization [6], resulting in substantial economic burden.

There is little data on actual prescribing practice for URTI in Asia. Evidence shows that symptomatic medications including antibiotics are of marginal or no benefit [7-13]. However, there are widespread variations in URTI management. A telephone survey of Auckland GPs found that $95 \%$ would, on occasions, prescribe asneeded medications (instructions for patient to fill prescriptions if condition worsens), including antibiotics [14]. In a European study, the number of medication types per patient varied from 0.82 to 3.55 per patient per year [15]. Interestingly, the antibiotic prescription rate in this European study did not vary with the overall medication prescription rate. A study in Zimbabwe highlighted widespread non-evidence based prescribing for URTI patients [16]. The actual prescribing practice among Chinese primary care physicians for URTI symptoms has not previously been investigated, although anecdotal observations show that medications for symptomatic relief are commonly used. Understanding the characteristics of patients who consult with URTI symptoms and the prescribing practices of primary care clinicians could identify opportunities for intervention such as reducing unnecessary prescriptions of antibiotic and symptomatic medication and reallocating resources for other medical conditions in primary care such as chronic disease management.

We therefore reviewed the characteristics of patients attending Hong Kong's public primary care clinics (PPCCs) with URTI symptoms, and the types of medications clinicians prescribed for URTI symptoms.

\section{Methods}

We performed a retrospective review of computerized clinical data on patients presenting to PPCCs with upper respiratory tract symptoms between $1^{\text {st }}$ January 2005 and $31^{\text {st }}$ December 2010. Upper respiratory tract symptoms were defined by specific ICPC (International Classification of Primary Care) codes agreed upon by an expert panel of local primary care physicians including:

- H71 - otitis media

- R72 - streptococcal pharyngitis

- R74 - acute upper respiratory tract infection

- R75 - acute sinusitis

- R76 - acute tonsillitis
- R77 - acute laryngitis

- R80 - influenza

There are 74 PPCCs in Hong Kong covering the entire population 7 million, with over 5 million attendances annually [17]. Consultations fees are subsidized for permanent residents (patient pays USD5.8 per consultation, inclusive of medications and investigations), while specific populations (government workers and those on financial assistance) receive free consultations. Existing literature suggests that less than 100,000 attendances are made to local emergency departments for non-urgent respiratory tract infections annually $[18,19]$, while $26 \%$ of private sector consultations (accounting for 50\% of Hong Kong's primary care) are related to respiratory tract infections [1].

Data on gender, age, chronic illness status, attendance date, type and duration of drug prescribed for URTI were extracted from the system-wide electronic record system (Clinical Data Analysis \& Reporting System [CDARS]). There is no information from CDARS for patients' financial status; nevertheless, those requiring financial assistance from the government can be identified separately from those paying the usual fee. Previous publications using CDARS have demonstrated the accuracy and completeness of the data retrieved from this system [20].

We defined a repeat attendance for the same URTI episode (which we considered as the same illness episode) as any attendances for URTI symptoms within 28 days of the previous URTI attendance. Medications were grouped into specific drug classes, including analgesia, antibiotics, antihistamines, cough syrup, mucolytic, $\mathrm{s} \alpha$-agonists, $\beta$-agonists, theophylline and other including throat lozenges, lysozyme.

All statistical analysis was performed using SPSS version 18.0 for Windows. Descriptive statistics were calculated using mean and range. Chi-square test was used to compare categorical variables across the different years. All $\mathrm{p}$ values $<0.05$ were regarded as statistically significant.

This study was approved by the Clinical Research Ethics Committee of the Chinese University of Hong Kong and New Territories East Cluster of the Hospital Authority.

\section{Results}

There were 5,529,755 attendances for RTIs from 2005 to 2010. Patient demographics and the conditions diagnosed are summarized in Table 1.

\section{Patient presentation}

There were 5,529,755 consultations for URTI symptoms in the study period for the patient population of $1,181,816$ [21]. The distribution of annual attendances was similar across the six years with the majority 
Table 1 Patient demographics and diagnosed conditions

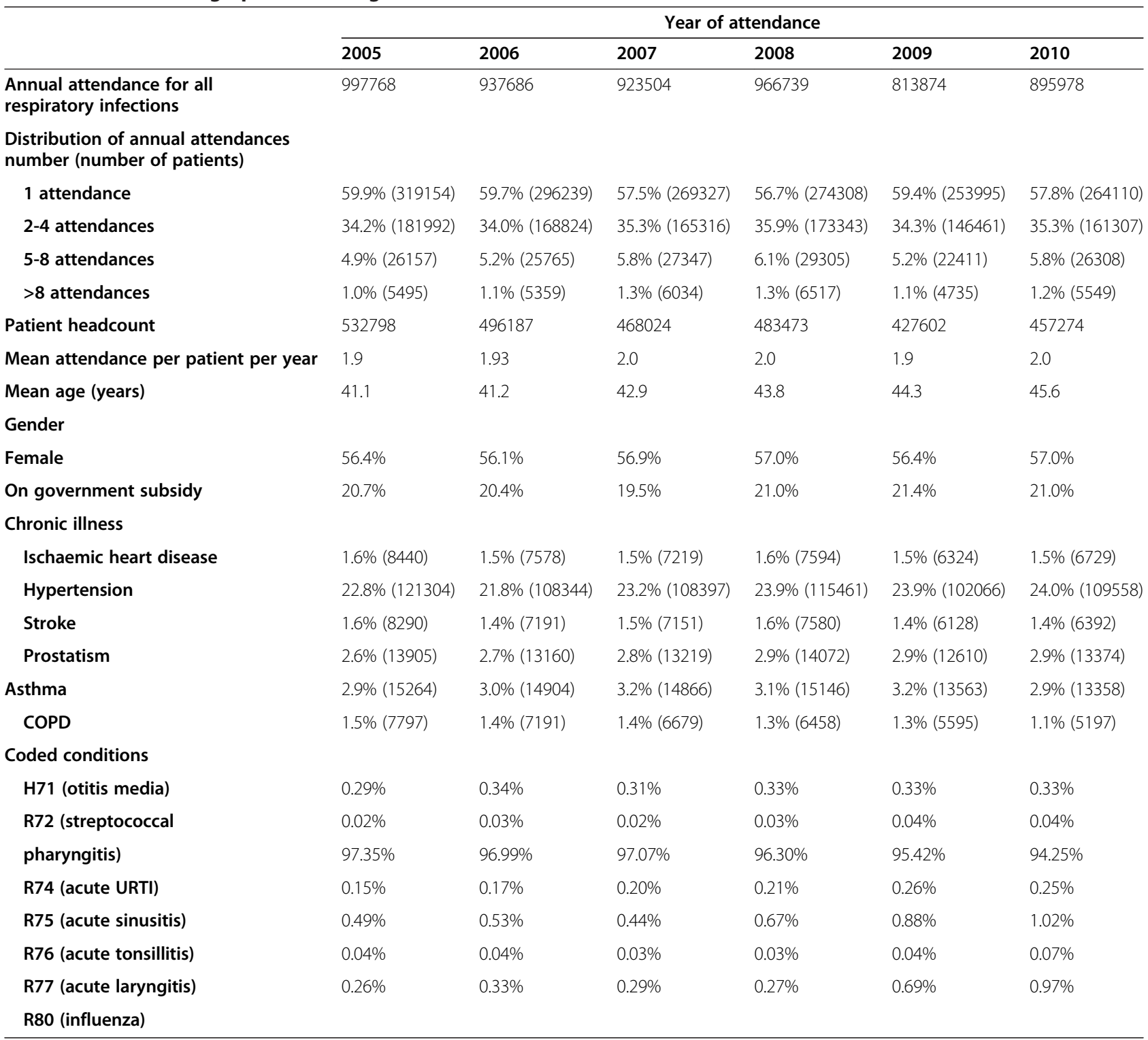

(approx 60\%) of patients presenting once for symptoms of upper respiratory tract infections. Over a third of the patients (approximately 35\%) attended 2-4 times per year. There was a 16\% drop in attendances in 2009. Overall, there was a significant increase $(p<0.001)$ in the mean number of annual attendances per patient.

The mean age of presentation shows a steady increase from 41.1 to 45.6 years from 2005 -2010. Patients were more likely to be female (approx $57 \%$ ) and on average one fifth of those presenting were on government subsidy. Over $94 \%$ were coded as upper respiratory tract infection (R74). Over the 6 years, there was a significant increase $(p<0.001)$ in the number of patients coded with non-R74 conditions.

\section{Prescription of medication}

Of the 5,529,755 primary care consultations for URTI symptoms from 2005 to 2010, 98\% resulted in a prescription of at least one medication (Table 2). Prescription patterns of symptomatic medication were largely similar across the 5 years, but there was a small but significant rise in the number of drugs given per consultation $(\mathrm{p}<0.001)$. The most commonly prescribed medications were sedating antihistamines (79.9\%), analgesia (58.9\%), throat lozenges $(40.4 \%)$ and expectorant cough syrup (33.8\%). During the study period, there was a significant decline in antibiotic prescription from $8.1 \%$ to $5.1 \%(\mathrm{p}<0.001)$. However, in consultations, where the given diagnosis was otitis media (H71), streptococcal 
Table 2 Details on drugs prescribed

\begin{tabular}{|c|c|c|c|c|c|c|c|}
\hline & & & Year of & endance & & & \\
\hline & 2005 & 2006 & 2007 & 2008 & 2009 & 2010 & \\
\hline Mean number of drugs & 3.1 & 3.1 & 3.2 & 3.2 & 3.2 & 3.2 & \\
\hline Number of drug items per prescription & & & & & & & \\
\hline 0 & $2.7 \%$ & $2.7 \%$ & $2.3 \%$ & $1.3 \%$ & $1.3 \%$ & $1.1 \%$ & \\
\hline 1 & $5.7 \%$ & $5.5 \%$ & $5.1 \%$ & $5.1 \%$ & $5.5 \%$ & $5.0 \%$ & \\
\hline 2 & $21.2 \%$ & $20.4 \%$ & $19.6 \%$ & $19.7 \%$ & $19.8 \%$ & $18.3 \%$ & \\
\hline 3 & $34.6 \%$ & $33.8 \%$ & $33.3 \%$ & $33.0 \%$ & $32.4 \%$ & $32.1 \%$ & \\
\hline 4 & $27.8 \%$ & $28.5 \%$ & $29.7 \%$ & $30.9 \%$ & $31.0 \%$ & $33.0 \%$ & \\
\hline$>4$ & $8.1 \%$ & $9.2 \%$ & $10.0 \%$ & $10.0 \%$ & $10.0 \%$ & $10.5 \%$ & \\
\hline Proportion of prescriptions containing & & & & & & & Current evidence \\
\hline Analgesics & & & & & & & \\
\hline Paracetamol & $58.3 \%$ & $60.1 \%$ & $59.8 \%$ & $59.1 \%$ & $58.7 \%$ & $58.9 \%$ & May reduce fever \\
\hline NSAIDs & $3.0 \%$ & $3.6 \%$ & $4.2 \%$ & $4.3 \%$ & $4.5 \%$ & $4.9 \%$ & May reduce pain \\
\hline COX-II inhibitors & $0.0 \%$ & $0.0 \%$ & $0.0 \%$ & $0.0 \%$ & $0.0 \%$ & $0.0 \%$ & No evidence \\
\hline Antibiotics & $8.1 \%$ & $8.1 \%$ & $6.8 \%$ & $4.9 \%$ & $4.4 \%$ & $5.1 \%$ & \\
\hline Antihistamines & & & & & & & \\
\hline Sedating & $81.1 \%$ & $80.1 \%$ & $82.3 \%$ & $82.4 \%$ & $80.8 \%$ & $79.9 \%$ & Ineffective \\
\hline Non-sedating & $0.5 \%$ & $0.3 \%$ & $0.3 \%$ & $0.3 \%$ & $0.6 \%$ & $1.2 \%$ & \\
\hline Cough syrup & & & & & & & \\
\hline Expectorant & $36.8 \%$ & $35.6 \%$ & $36.3 \%$ & $36.3 \%$ & $34.0 \%$ & $33.8 \%$ & Not recommended in children \\
\hline Codeine & $7.1 \%$ & $6.9 \%$ & $6.7 \%$ & $6.5 \%$ & $6.6 \%$ & $6.3 \%$ & Limited evidence \\
\hline Mucolytic & $20.9 \%$ & $22.2 \%$ & $23.0 \%$ & $24.0 \%$ & $24.0 \%$ & $25.0 \%$ & May reduce symptoms \\
\hline Oral a-agonist & $0.6 \%$ & $0.4 \%$ & $0.3 \%$ & $0.2 \%$ & $0.2 \%$ & $0.2 \%$ & Modest effect in adults \\
\hline Oral $\beta$-agonist & $4.4 \%$ & $4.7 \%$ & $4.2 \%$ & $4.1 \%$ & $3.7 \%$ & $4.1 \%$ & No evidence \\
\hline Theophylline & $1.1 \%$ & $1.0 \%$ & $1.0 \%$ & $0.9 \%$ & $0.8 \%$ & $0.9 \%$ & No evidence \\
\hline Others & & & & & & & \\
\hline Throat lozenges & $34.7 \%$ & $34.9 \%$ & $35.6 \%$ & $37.5 \%$ & $39.9 \%$ & $40.4 \%$ & No evidence \\
\hline Lysozyme & $10.6 \%$ & $11.8 \%$ & $13.8 \%$ & $14.5 \%$ & $14.9 \%$ & $15.6 \%$ & No evidence \\
\hline Neozep & $0.6 \%$ & $0.3 \%$ & $0.3 \%$ & $0.2 \%$ & $0.0 \%$ & $0.1 \%$ & \\
\hline
\end{tabular}

pharyngitis (R72), acute sinusitis (R75) and acute laryngitis (R76), over 90\% resulted in antibiotic prescription (Table 3 ). Almost $50 \%$ of patients diagnosed with influenza were prescribed antibiotics. The proportion was much less among those coded with R74 (acute URTI).

\section{Discussion}

\section{Main study findings}

Consultations for URTI symptoms account for almost $20 \%$ of attendances per year in Hong Kong's public primary care (around 900,000 out of 5 million attendances

Table 3 Proportion of antibiotic prescribed in different URTIs

\begin{tabular}{lccccccc}
\hline & \multicolumn{7}{c}{ Condition } \\
\cline { 2 - 7 } Year & $\mathbf{H 7 1}$ & $\mathbf{R 7 2}$ & $\mathbf{R 7 4}$ & $\mathbf{R 7 5}$ & $\mathbf{R 7 6}$ & $\mathbf{R 7 7}$ & $\mathbf{R}$ \\
\hline $\mathbf{2 0 0 5}$ & $94.4 \%(2816)$ & $89.2 \%(213)$ & $6.5 \%(957615)$ & $90.7 \%(1523)$ & $95.2 \%(4776)$ & $23.9 \%(348)$ & $54.0 \%(2556)$ \\
$\mathbf{2 0 0 6}$ & $95.4 \%(3135)$ & $80.6 \%(294)$ & $6.3 \%(897381)$ & $86.1 \%(1529)$ & $96.0 \%(4915)$ & $25.4 \%(378)$ & $52.8 \%(3087)$ \\
$\mathbf{2 0 0 7}$ & $95.2 \%(2798)$ & $81.7 \%(164)$ & $5.1 \%(889038)$ & $85.0 \%(1788)$ & $94.7 \%(4005)$ & $26.9 \%(283)$ & $50.6 \%(2669)$ \\
$\mathbf{2 0 0 8}$ & $94.1 \%(3179)$ & $93.9 \%(245)$ & $2.6 \%(937252)$ & $91.6 \%(2024)$ & $96.7 \%(6543)$ & $59.1 \%(281)$ & $47.0 \%(2623)$ \\
$\mathbf{2 0 0 9}$ & $94.4 \%(2683)$ & $95.5 \%(352)$ & $1.8 \%(782692)$ & $92.0 \%(2138)$ & $97.8 \%(7233)$ & $68.6 \%(338)$ & $43.1 \%(5697)$ \\
$\mathbf{2 0 1 0}$ & $94.4 \%(3044)$ & $97.8 \%(320)$ & $1.9 \%(858979)$ & $91.3 \%(2317)$ & $97.9 \%(9317)$ & $73.6 \%(658)$ & $48.2 \%(8882)$ \\
\hline
\end{tabular}

Bracketed values indicate the number of attendances where the specified ICPC code was assigned. 
annually [17]). There was a drop in attendances in 2009, which coincides with the swine flu outbreak that year, where patients with influenza like illness were advised to go to designated fever clinics. There is an increasing mean age of patients presenting to primary care for upper respiratory tract infections which may reflect the increasing age of the population and those presenting to primary care. $98 \%$ of consultations were associated with a prescription, which on average included four drug classes. Antibiotics were prescribed in $5 \%$ of consultations.

\section{Antibiotic prescribing}

The overall antibiotic prescription rate observed in this study was much lower than in other countries [22-25]. Although patients with uncomplicated URTIs (R74) comprise the large majority of the population, it appears that the overall volume of antibiotics prescribed remains low. However, nearly all those coded with H71 (otitis media), R72 (streptococcal pharyngitis), R75 (acute sinusitis) and R76 (acute tonsillitis) were almost always prescribed antibiotics, with an increasing trend among R72 and R77. Furthermore, around $50 \%$ of those diagnosed with influenza (R80) were also prescribed with antibiotics. Prescribing pressure [26] from patients may be an issue, especially among those with more troubling symptoms. This could also reflect clinicians' 'coding creep' or 'diagnostic drift' with the tendency to use non-R74 codes in consultations where antibiotics were prescribed. Further education and training in terms of diagnosis, management and use of correct coding may need to be instated. Whilst financial incentive is an important contributory factor for 'coding creep' in some countries [27-29], this is not an issue in Hong Kong where there is no pay-for-performance system in place. Further education of doctors in the appropriate management of otitis media, streptococcal pharyngitic, acute sinusitis and acute tonsillitis may help to reduce unnecessary antibiotic prescription.

\section{Symptomatic drug utilization}

At least 3 medications are prescribed for each URTI episode. This is higher than the URTI drug prescription patterns of Dutch doctors, where a mean of 0.8 drugs were prescribed per patient. Possible explanations for a higher prescription number may be related to the nature of the Hong Kong healthcare system, where consultation fees in PPCCs are heavily subsidized and includes the cost of medication, patients' relatively low self medication rate [30] and high expectations for receiving a prescription for medication during consultations [31]. The slight increase in the number of medications prescribed coincided with an increase in annual attendances per patient for URTI, suggesting that this prescribing habit may be encouraging patients to consult. Although one previous European study indicated that medication prescribing rates did not vary with antibiotic prescription rate [15], further research would be warranted in view of the overall low antibiotic prescription volume.

A large variety of symptomatic medications were prescribed, including $\alpha$ and $\beta$-agonists, lysozyme and mucolytics. There is little empirical evidence that these medicines are effective for URTI [32-35]. Anecdotally, it is not uncommon for patients to request a medication for each symptom they experience, which may help explain why patients are prescribed antitussives, antihistamines, mucolytics and throat lozenges. Among these prescriptions, $80 \%$ were on sedating antihistamines; $6 \%$ were given opioid containing medications; and a minority was prescribed theophyllines, $\alpha$-agonists or $\beta$-agonists. There is a need for further research on the expectations of symptomatic medication prescriptions as recipients of these medications included children, and patients with chronic disease in which medication side effects could be detrimental e.g. cardiac diseases, prostatic symptoms or respiratory problems [36-39].

\section{Implication for health service provision and future research}

Compared with European countries [40-43], Hong Kong's public primary care appears to exhibit low antibiotic prescribing behaviour for symptoms of upper respiratory tract infections. On the other hand, the prescription rate for non-antibiotic medications is exceptionally high. It may be that prescription of symptomatic medication has managed to displace the expectation of antibiotic prescription. However, symptomatic drug prescription is high, and is estimated to cost the public healthcare system over 1.2 million US dollars per year. Other costs including PPCCs' facilities and human resources, as well as the societal impact of health services utilization for URTI have not been included.

Previous studies have shown that a significant proportion of primary care physicians believe patients should be empowered to self manage uncomplicated URTI [44]. Nevertheless, many patients continue to consult doctors within the first two days of their illnesses [30,31] despite its self-limiting nature. Public health initiatives are therefore needed in order to promote self-management and appropriate indications for help seeking. The gap between clinical evidence for pharmacological intervention, doctors' prescribing behavior and patient expectation must also be addressed in order to safeguard patient wellbeing and the appropriate use of health care resources. Primary care physicians can play an active role to empower patients to self-manage symptoms and in reducing the reliance on unnecessary medications whenever appropriate. Further studies looking into cultural differences in expectations and management of URTI like 
symptoms can better inform effective regional educational interventions.

\section{Strengths and limitations of this study}

This is the first study of patient characteristics and prescribed medication among patients presenting to public primary care clinics with URTIs in the South China Region. The availability of electronic patient data from all PPCCs in Hong Kong ensured coverage of all patients in the public primary care setting, allowing an accurate analysis into the health services utilization related to URTIs. Internal audits within the Hospital Authority (unpublished data) have indicated that the ICPC coding rate among PPCCs is generally over $90 \%$. Two studies examining ICPC coding accuracy over the recent few years $[45,46]$ have shown that $96 \%$ to $98 \%$ of cases are coded accurately, especially if the case is not complex. These imply that the data obtained for this study accurately reflects the public primary care situation in our locality.

Those who utilize public clinics in primary care are more likely to be of lower socio-economic status, elderly and individuals with chronic conditions [47], this is reflected in our study where one fifth is on financial assistance and the increasing age of presentation. However, results do not include consultations in the private sector which is likely to see patients who are more affluent and younger. Meanwhile this study has important implications as older people are more prone to drugdrug interactions and adverse events from the prescriptions of medications for URTI. Moreover, as there is a high rate of 'doctor shopping' among patients in primary care in Hong Kong, this pattern of high utilization of symptomatic prescriptions for URTI in Hong Kong is likely to be common among most patients consulting primary care in Hong Kong, both in the private sector and in government run clinics given similar expectations.

\section{Conclusions}

Overall the rate of antibiotic prescription for patients attending Hong Kong's public primary care is low, although the rate is high among those diagnosed with otitis media, pharyngitis, sinusitis and tonsillitis. On the other hand, the number symptomatic medications prescribed is high, and its relationship with increasing doctor consultations and low overall antibiotic prescription need to be further explored. Gaps have been identified between primary care practice and evidence based management of upper respiratory tract infections. There is a huge potential to save resources on inappropriate antibiotic use, unnecessary prescription of symptomatic medication and consultation appointments through doctor and patient education.
Competing interests

The authors declare that they have no competing interests.

\section{Author contributions}

KK/CW conceptualised the whole study. KK researched data and wrote the manuscript, with further analysis performed by CKYC. CW/SYSW/AL/SG/CCB reviewed the manuscript. CCB contributed to discussion and reviewed the manuscript. All authors declare that there are no financial relationships with any organisations that might have an interest in the submitted work, and that no presentations have been made for this research. All authors read and approved the final manuscript.

\section{Acknowledgements}

This work was supported by the primary care team at the Chinese University of Hong Kong, without contribution from any external funding sources.

\section{Author details}

'Division of Family Medicine, School of Public Health \& Primary Care, Chinese University of Hong Kong, Shatin, Hong Kong. ${ }^{2}$ Department of Family Medicine, the Hospital Authority, Kowloon, Hong Kong. ${ }^{3}$ Department of General Practice, Cardiff University, Cardiff, UK. ${ }^{4}$ Jockey School of Public Health, Prince of Wales Hospital, Room 408, 32 Ngan Shing Street, Shatin, NT, Hong Kong.

Received: 13 January 2014 Accepted: 29 April 2014

Published: 13 May 2014

\section{References}

1. Lo Y, Lam C, Lam T, Lee R, Chiu B, Tang J, Chui B, Chao D, Lam A, Chan K: Hong Kong primary care morbidity survey 2007-2008. HK Pract 2010, 32:17-26.

2. Hak E, Rovers M, Kuyvenhoven M, Schellevis F, Verheij T: Incidence of GP-diagnosed respiratory tract infections according to age, gender and high-risk co-morbidity: the Second Dutch National Survey of General Practice. Fam Pract 2006, 23(3):291-294.

3. Fleming D, Smith G, Charlton J, Charlton J, Nicoll A: Impact of infections on primary care - greater than expected. Commun Dis Public Health 2002, 5(1):7-12.

4. Lam W, Ho K, Kwok K, Tsang L: Morbidity pattern in four government general practice clinics using the International Classification of Primary Care (Revised Edition) (ICPC-2) coding. HK Pract 2006, 28:363-375.

5. CDARS: Clinical Decision Analysis Reporting System. Hong Kong: Department of Family Medicine, New Territories East Cluster, Hospital Authority. Last accessed 12 Jul 2011

6. Nichol K, D'Heilly S, Ehlinger E: Burden of upper respiratory illnesses among college and university students: 2002-2003 and 2003-2004 cohorts. Vaccine 2006, 24(44-46):6724-6725

7. Taverner D, Latte J: Nasal decongestants for the common cold [update of Cochrane Review]. 2007, (1):CD001953.

8. Kernan W, Viscoli C, Brass L: Phenylpropanolamine and the risk of hemorrhagic stroke. N Engl J Med 2000, 343:1826-1832.

9. Arroll B, Kenealy T: Are antibiotics effective for acute purulent rhinitis? Systematic review and meta-analysis of placebo controlled randomised trials. BMJ 2007, 333:279.

10. Fahey T, Stocks N, Thomas T: Systematic review of the treatment of upper respiratory tract infection. Arch Dis Child 1998, 79:225-230.

11. Sutter Al, Lemiengre M, Campbell H, Mackinnon HF: Antihistamines for the common cold. Cochrane Database Syst Rev 2009, (4):CD001267.

12. Yoder K, Shaffer M, La Tournous S: Child assessment of dextromethorphan, diphenhydramine, and placebo for nocturnal cough due to upper respiratory infection. Clin Pediatr 2006, 45:633-640.

13. Lam C: Rational prescribing for upper respiratory tract infections. HK Pract 2002, 24:298-304.

14. Arroll B, Goodyear-Smith F: General practitioner management of upper respiratory tract infections: when are antibiotics prescribed? NZJM 2000, 493-496.

15. Rosman S, Le Vaillant M, Schellevis F, Clerc P, Verheij R, Pelletier-Fleury N: Prescribing patterns for upper respiratory tract infections in general practice in France and in the Netherlands. Eur J of Pub Health 2008, 18(3):312-316 
16. Trap B, Hansen E: Treatment of upper respiratory tract infections - a comparative study of dispensing and non-dispensing doctors. J Clin Pharma Therapeut 2002, 27:289-298.

17. Statistics \& Workforce Planning Department, Strategy \& Planning Division, Hospital Authority Head Office: GOP Service Portfolio 2012. Hong Kong Hospital Authority; 2012.

18. Lee A, Lau F, Hazelett C, Kam C, Wong P, Wong TW, Chow S: Morbidity patterns of non-urgent patients attending accident and emergency departments in Hong Kong: cross-sectional study. HKMJ 2001, 7:131-138

19. Law C, Yip P: Acute care service utilisation and the possible impacts of a user-fee policy in Hong Kong. HKMJ 2002, 8:348-353.

20. Wong $M$, Jiang J, Tang J, Lam A, Fung H, Mercer S: Health services research in the public healthcare system in Hong Kong: An analysis of over 1 million antihypertensive prescriptions between 2004-2007 as an example of the potential and pitfalls of using routinely collected electronic patient data. BMC Health Serv Res 2008, 8:138.

21. Census and Statistics Department: The Government of the Hong Kong Special Administration Region. Population Census; 2006. http://www.statistics.gov.hk/ pub/B11200512006XXXXB0400.pdf. Last accessed 8 May 2014.

22. Cheong L, Kwok C, Aljunid S, Cheah M: Antibiotic prescription in upper respiratory tract infections. Asia Pacfici Family Medicine 2004, 3(1-2):38-45.

23. Gonzales R, Steiner J, Sande M: Antibiotic prescribing for adults with colds, upper respiratory tract infections, and bronchitis by ambulatory care physicians. JAMA 1997, 278(11):901-904.

24. Hui L, Li X, Zeng X, Dai Y: Patterns and determinants of use of antibiotics for acute respiratory tract infection in children in China. Paed Infect Dis $J$ 1997, 16(6):560-564.

25. Arustiyono: Promoting rational use of drugs at the community health centres in Indonesia. WHO World Bank Health Project IV; 1999. http://archives.who.int/ prduc2004/Resource_Mats/Asia_papers/PROMOTING\%20RATIONAL\%20USE\% 200F\%20DRUGS\%20IN\%20INDONESIA.htm. Last accessed 8 May 2014.

26. Stivers T: Prescribing under pressure: parent-physician conversations and antibiotics. Oxford; New York: Oxford University Press; 2007:221.

27. Pongpirul K, Walker DG, Rahman H, Robinson C: DRG coding practice: a nationwide hospital survey in Thailand. BMC Health Serv Res 2011, 11(1):290.

28. Simborg DW: DRG Creep: A New Hospital-Acquired Disease. N Engl J Med 1981, 304(26):1602-1604

29. Agrawal S: Educating Physicians About Responsible Management of Finite Resources. JAMA 2013, 309(11):1115.

30. Lee A, Siu S, Lam A, Tsang C, Kung K, Li P: The concepts of family doctor and the factors affecting choice of family doctors among Hong Kong people. Hong Kong Med J 2010, 16:18-25.

31. Chan C: What do patients expect from consultations for upper respiratory tract infections? Fam Pract 1996, 13:229-235.

32. Duijvestijn Y, Mourdi N, Smucny J, Pons G, Chalumeau M: Acetylcysteine and carbocysteine for acute upper and lower respiratory tract infections in paediatric patients without chronic broncho-pulmonary disease. Cochran Database Syst Rev 2009, 1:CD003124.

33. Smith S, Schroeder K, Fahey T: Over-the-counter (OTC) medications for acute cough in children and adults in ambulatory settings. Cochran Database Syst Rev 2008, 1:CD001831.

34. Kim S, Chang Y, Cho H, Hwang Y, Moon Y: Non-steroidal antiinflammatory drugs for the common cold. Cochran Database Syst Rev 2009, 3:CD006362

35. Arroll B: Respiratory disorders (acute) - common cold. Clinical Evidence 2011, 03:1510.

36. Hong Kong Department of Health, Pharmaceutical Registration Section: Drug alert on the use of oral liquid cough medicines containing codeine. Hong Kong: Department of Health; 2010.

37. Church M, Maurer M, Simons F, Bindslev-Jensen $C$, van Cauwenberge $P$, Bousquet J, Holgate ST, Zuberbier T: Risk of first-generation H1- antihistamines: a GA2LEN position paper. Allergy 2010, 65:459-466.

38. $\mathrm{Ng}$ D: Prescription of codeine in young infants. Hong Kong Med J 2004 10(6):439.

39. Medicines and Healthcare Products Regulatory Agency: Oral liquid cough medicines containing codeine: should not be used in children and young people under 18 years. MHRA Public Assessment Report; 2010. http://www. mhra.gov.uk/home/groups/pl-p/documents/websiteresources/con096798. pdf. Last accessed 8 May 2014.
40. Carrie AG, Zhanel GG: Antibacterial use in community practice: assessing quantity, indications and appropriateness, and relationship to the development of antibacterial resistance. Drugs 1999, 57(6):871-881.

41. McGregor A, Dovey S, Tilyard M: Antibiotic use in upper respiratory tract infections in New Zealand. Fam Pract 1995, 12(2):166-170.

42. Fagnani F, German-Fattal M: Antibiotic prescribing patterns of French GPs for upper respiratory tract infections: impact of fusafungine on rates of prescription of systemic antibiotics. Am J Respir Med 2003, 2(6):491-498.

43. Ashworth M, Cox K, Latinovic R, Charlton J, Gulliford M, Rowlands G: Why has antibiotic prescribing for respiratory illness declined in primary care? A longitudinal study using the General Practice Research Database. J Public Health 2004, 26(3):268-274.

44. Lam T, Lam K: Family doctors' attitudes towards patient selfmanagement of upper respiratory tract infections. Hong Kong Med J 2001, 7:146-149.

45. Wong $\mathrm{H}$, Chan $\mathrm{E}$, Wong $\mathrm{M}$, Liang J: Is the number of clinical problems per consultation correlated with poorer ICPC coding practices in primary care clinics? A pilot study in two distinct clinic settings. HK Pract 2009 31:158-165.

46. Wong L, Lee M, Mak H, Wong K, Chung K, Wong K, Tam S, Li H, Chan T, Kung K, Lam A, Li P: Accuracy and completeness of ICPC coding for chronic disease in general outpatient clinics. HK Pract 2010, 32:129-135.

47. Wong S, Kung K, Griffiths S, Carthy T, Wong M, Lo S, Chung V, Goggins W, Starfield B: Comparison of primary care experiences among adults in general outpatient clinics and private general practice clinics in Hong Kong. BMC Public Health [Internet] 2010, 10:397. Available from: http://www.biomedcentral. com/1471-2458/10/397.

doi:10.1186/1471-2296-15-95

Cite this article as: Kung et al:: Patient presentation and physician management of upper respiratory tract infections: a retrospective review of over 5 million primary clinic consultations in Hong Kong. BMC Family Practice 2014 15:95.

\section{Submit your next manuscript to BioMed Central and take full advantage of:}

- Convenient online submission

- Thorough peer review

- No space constraints or color figure charges

- Immediate publication on acceptance

- Inclusion in PubMed, CAS, Scopus and Google Scholar

- Research which is freely available for redistribution 